\title{
Simplified Fokker-Plank Equation Treatment of Finite-temperature Spin-torque Problems
}

\author{
Xia Jianbai", Wen Hongyu \\ Institute of Semiconductors, University of Chinese Academy of Sciences, Beijing, China \\ Email address: \\ xiajb@semi.ac.cn (Xia Jianbai), wenhongyu@semi.ac.cn (Wen Hongyu) \\ ${ }^{*}$ Corresponding author
}

To cite this article:

Xia Jianbai, Wen Hongyu. Simplified Fokker-Plank Equation Treatment of Finite-temperature Spin-torque Problems. American Journal of Physics and Applications. Vol. 7, No. 2, 2019, pp. 55-60. doi: 10.11648/j.ajpa.20190702.14

Received: January 11, 2019; Accepted: May 23, 2019; Published: June 12, 2019

\begin{abstract}
A Legendre function expansion method is proposed to solve the simplified Fokker-Plank equation to study the dynamics of a macrospin under spin-torque-driven magnetic reversal at finite temperature. The first and second eigenvalues $\left(\lambda \tau_{0}\right)_{1}$ and $\left(\lambda \tau_{0}\right)_{2}$ as functions of $I / I c$ and $H_{k}$ are obtained, in agreement with the previous results using the Taylor series expansion method. The Legendre function expansion method compared with the Taylor series expansion method has faster convergence properties and clear physical means. Besides, it is found that in some case, especially the second eigenvalue $\left(\lambda \tau_{0}\right)_{2}$ can become complex, that means that the probability density $P$ not only decays with time, but also oscillates with time.
\end{abstract}

Keywords: Fokker-Plank Equation, Legendre Function, Thermal Fluctuation, Magnetic Reversal

\section{Introduction}

In the spin-torque-induced switching the write and read time is essential. [1-3] There are two aspects which will affect the reversal time $\tau_{s}$. First, the initial position of the magnetic moment is thermally distributed at the time the reversal field or current is applied, causing a variation in switching time. Secondly, during reversal, thermal fluctuation would modify the orbit, [4-7] causing additional fluctuation for $\tau_{s}$ even for identical initial conditions.

The dynamics of a macrospinunder spin-torque-driven magnetic reversal has been extensively studied. [8-9] In the limit of uniaxial anisotropy only and with finite temperature at large drive amplitude $I \gg>I_{c 0}$, with $I$ being the current passing through the junction, $I_{c 0}$ the zero-temperature spin-torque current instability threshold, the "long-time" super-threshold asymptotic form for the probability of not switching at time $t$ can be expressed as:

$$
E_{r}(t) \approx\left(\frac{\pi^{2} \xi}{4}\right) \exp \left(-\frac{2 t}{\tau_{I}}\right)+O\left[\exp \left(-\frac{\pi^{2} \xi}{4}\right)\right]
$$

(for $I>>I_{c 0}, E_{r}<<1$ and $\xi>>1$ ) where $\xi=m H_{k} / 2 k_{B} T$ is the normalized thermal activation energy barrier height, $m$ is the total magnetic moment of the free layer, and $H_{k}$ the uniaxial anisotropy field. $\tau_{I}=\tau_{0} /\left(I / I_{c 0}-1\right)$ is the characteristic time scale for spin-torque-induced reversal, $\tau_{0}=1 / \mathcal{H}_{k} \alpha$ is the natural unit of time with $\gamma \approx 2 \mu_{B} / \hbar$ as the gyromagnetic constant, and $\alpha$ the LLG damping coefficient. The comparison of Eq. (1) with experimental results suggests the presence of sub volume magnetic excitations which often dominate the switching process and which degrade the spin-torque switching efficiency. [9]

He et al. presented a Fokker-Plank formulation for the full problem including both thermalized initial condition and reversal orbit with estimates for the reversal time and its distribution. [10] In the case of uniaxial anisotropy they reduced the Fokker-Plank equation (FPE) to an ordinary differential equation in which the lowest eigenvalue $\lambda_{1}$ determines the slowest switching events. They calculated $\lambda_{1}$ using both analytical and numerical methods. It is found that the previous model [Eq. (1)] based on thermally distributed initial magnetization states can be accurately justified in some useful limiting conditions.

In this paper we use the simplified FPE to study the switch time at the finite temperature by solving the eigenvalue equation derived from FPE. 


\section{Theoretical Demonstration and Formula Derivation}

\subsection{Simplified Fokker-Plank Equation}

Fokker-Plank equation describes the time-dependent evolution of the ensemble-average probability distribution of a system in a given environment and initial condition. [10] For a macrospin, one defines a probability density function $P\left(n_{m}\right.$, $t)=P(\theta, \varphi, t)$ that is the time-dependent probability of finding the macrospin in the solid angle of $\sin \theta d \theta d \varphi$ with a spherical coordinate set $(\theta, \varphi)$ describing the magnet's direction, $n_{m}$. The Fokker-Plank equation describes the dynamic flow of this probability as a function of space (on the surface of a unit sphere) and time in the form of:

$$
\frac{\partial P}{\partial t}+\nabla \cdot \boldsymbol{J}-D \nabla^{2} P=0
$$

where

$$
\boldsymbol{J}=P \frac{d \boldsymbol{n}_{m}}{d t}
$$

is the ballistic (zero-temperature) part of the probability current and $D \nabla^{2} P=\nabla \cdot J_{D}$ is the diffusive part of the probability current, with $J_{D}=D \nabla P$. The constant $D$ is the diffusion rate in the probability phase space, $D=\gamma \alpha k_{B} T / m$ [10]. $d n_{m} / d t$ is determined by the LLG equation including the spin-torque term,

$$
\frac{1}{\gamma}\left(\frac{d \boldsymbol{n}_{m}}{d t}+\alpha \boldsymbol{n}_{m} \times \frac{d \boldsymbol{n}_{m}}{d t}\right)=\boldsymbol{n}_{m} \times \boldsymbol{H}_{e f f}+\left(\frac{I_{s}}{m}\right) \boldsymbol{n}_{m} \times\left(\boldsymbol{n}_{m} \times \boldsymbol{n}_{s}\right)
$$

where $n_{s}$ is the unit vector of the magnetization direction in the pinned layer. Eqs. (1), (2) and (3) give a set of partial equations that describes the magnetic moment dynamics at the finite temperature in the spin-torque condition. The problem can be solved numerically in principle, here we refer a simplified solution of the set of Fokker-Plank equations [11].

Consider an ensemble time-dependent magnetization probability density $P\left(n_{m}, t\right)$. The unit vector of magnetic moment $n_{m}$ is characterized by the polar angle $(\theta, \phi)$. Before the magnetic field and the current are turned on, the probability density takes the equilibrium value. For a uniaxial anisotropy situation,

$$
P\left(\boldsymbol{n}_{m}, 0\right)=\left\{\begin{array}{cc}
P_{0} \exp \left(-\xi \sin ^{2} \theta\right), & 0 \leq \theta \leq \pi / 2 \\
0, & \pi / 2<\theta \leq \pi
\end{array}\right.
$$

where $P_{0}$ is the normalization factor, determined by.

$$
\int_{0}^{\pi} P \sin \theta d \theta=1
$$

The probability current is derived by the LLG Eq. (4),

$$
\boldsymbol{J}=P \frac{d \boldsymbol{n}_{m}}{d t}=-\gamma P \boldsymbol{n}_{m} \times\left[\boldsymbol{H}_{e}+\alpha \boldsymbol{n}_{m} \times\left(\boldsymbol{H}_{e}-\boldsymbol{H}_{s}\right)\right]
$$

where $H_{s}$ is the spin torque term,

$$
\boldsymbol{H}_{s}=\operatorname{Ip}\left(\frac{\hbar}{2 q}\right)\left(\frac{1}{\alpha m}\right) \boldsymbol{n}_{s}
$$

$I$ is the current density, $p$ is the spin polarization coefficient, $q$ is the electron charge. In the simplified treatment of the Fokker-Plank equation [10], it is assumed that the magnetic field $H$ and $n_{s}$ are parallel to the anisotropy axis, i.e., $H_{e}=$ $\left(H+H_{k} \cos \theta\right) e_{z}$ and $n_{s}=e_{z}$ where $H_{k}=2 K / M_{s}$.

In the polar coordinate,

$$
\nabla \cdot J=\frac{1}{r^{2}} \frac{\partial}{\partial r}\left(r^{2} J_{1}\right)+\frac{1}{r \sin \theta} \frac{\partial}{\partial \theta}\left(\sin \theta J_{2}\right)+\frac{1}{r \sin \theta} \frac{\partial J_{3}}{\partial \phi}
$$

From Eq. (6) $J$ has only $e_{\theta}$ and $e_{\phi}$ components, i.e. $J_{2}$ and $J_{3}$, $J_{1}=0$.

$$
\begin{aligned}
& J_{2}=-\gamma P \alpha\left(H_{e}-H_{s}\right) \\
& J_{3}=\gamma P H_{e}
\end{aligned}
$$

Due to the axial symmetry $J_{2}$ and $J_{3}$ are only functions of $\theta$, and let.

$$
U_{e}=\left(H_{s}-H\right) m x-\left(\frac{H_{k}}{2}\right) m x^{2}
$$

We obtain the second term in Eq. (1),

$$
\nabla \cdot J=-\frac{\alpha \gamma}{m} \frac{\partial}{\partial x}\left[\left(1-x^{2}\right) \frac{\partial U_{e}}{\partial x} P\right]
$$

where $x=\cos \theta$.

In the polar coordinate,

$$
\nabla^{2} P=\frac{1}{r^{2}}\left(\frac{\partial}{\partial r} r^{2} \frac{\partial P}{\partial r}\right)+\frac{1}{r^{2} \sin \theta} \frac{\partial}{\partial \theta}\left(\sin \theta \frac{\partial P}{\partial \theta}\right)+\frac{1}{r^{2} \sin ^{2} \theta} \frac{\partial^{2} P}{\partial \phi^{2}}
$$

Because $P$ is only function of $\theta$, we obtain the third term in Eq. (1),

$$
D \nabla^{2} P=\frac{\alpha \gamma}{m} \frac{\partial}{\partial x}\left[\left(1-x^{2}\right) k_{B} T \frac{\partial P}{\partial x}\right]
$$

Then Eq. (1) reduces to.

$$
\frac{\partial P}{\partial t}=\frac{\alpha \gamma}{m} \frac{\partial}{\partial x}\left[\left(1-x^{2}\right)\left(\frac{\partial U_{e}}{\partial x} P+k_{B} T \frac{\partial P}{\partial x}\right)\right]
$$

Eq. (14) can be solved by the method of separation of 
variables. Let $P(x, t)=f(t) u(x)$, it is easy to see that $f(t)=$ $\exp (-\lambda t)$ and $u(x)$ satisfies.

$$
\frac{\alpha \gamma}{m} \frac{d}{d x}\left[\left(1-x^{2}\right)\left(\frac{d U_{e}}{d x} u(x)+k_{B} T \frac{d u(x)}{d x}\right)\right]=-\lambda u(x)
$$

Let.

$$
F(x)=e^{\beta U_{e}(x)} u(x), \quad c=\frac{\lambda m}{\alpha \gamma k_{B} T}
$$

Eq. (15) can be written as.

$$
e^{\beta U_{e}} \frac{d}{d x}\left[\left(1-x^{2}\right) e^{-\beta U_{e}} \frac{d F}{d x}\right]=-c F
$$

The original Fokker-Plank equation (2) is now reduced to the standard eigenvalue problem. If determining eigenfunction $F(x)=F_{n}(x)$ and eigenvalue $\lambda=\lambda_{n}$ from Eq. (17) for $n=1,2$, $3, \ldots$, and the general solution of Eq. (14) is.

$$
P(x, t)=\sum_{n=1} A_{n} e^{-\beta U_{e}(x)} F_{n}(x) e^{-\lambda_{n} t}
$$

where the coefficients in Eq. (18) are determined by the initial condition Eq. (5),

$$
A_{n}=\int_{0}^{1} d x P(x, 0) F_{n}(x)
$$

Expand the function $F(x)$ in Eq. (17) with the Legendre functions $P_{m}(x)$ defined in the region [-1, 1], [12]

$$
F(x)=\sum_{m=1} D_{m} P_{m}(x)
$$

Insert Eq. (20) into Eq. (17), multiply $p_{n}(x)$ on the both sides, and integrate $x$ from -1 to 1 . From the right side it obtains.

$$
-c D_{n} \frac{2}{2 n+1} \delta_{n m}
$$

from the left side it obtains the integration,

$$
-\int_{-1}^{1}\left(1-x^{2}\right) e^{-\beta U_{e}} \frac{d F}{d x} \frac{d}{d x}\left[e^{\beta U_{e}} P_{n}\right] d x
$$

which includes two terms. The first term (differs from a

$$
\begin{aligned}
& \int_{-1}^{1} d x \frac{m(m+1)}{2 m+1}\left(P_{m-1}-P_{m+1}\right)\left[\frac{\eta(n+1)}{2 n+1} P_{n+1}+\zeta P_{n}+\frac{\eta n}{2 n+1} P_{n-1}\right] \\
& =\frac{m(m+1)}{2 m+1}\left[\frac{\eta(n+1)}{2 n+1} \frac{2}{2 n+3} \delta_{m-2, n}+\zeta \frac{2}{2 n+1} \delta_{m-1, n}+\frac{\eta n}{2 n+1} \frac{2}{2 n-1} \delta_{m, n}\right. \\
& \left.-\frac{\eta(n+1)}{2 n+1} \frac{2}{2 n+3} \delta_{m, n}-\zeta \frac{2}{2 n+1} \delta_{m+1, n}-\frac{\eta n}{2 n+1} \frac{2}{2 n-1} \delta_{m+2, n}\right]
\end{aligned}
$$

By use of the paper [12].

Eq. (24) becomes.

From Eq. (10),

and by use of the paper [12].

Eq. (26) becomes.

By use of the paper [11].

Finally the integration (29) becomes.

$$
\frac{d}{d x}\left[\left(1-x^{2}\right) P_{m}^{\prime}\right]=-m(m+1) P_{m}
$$

$$
\sum_{m} D_{m} m(m+1) \int_{-1}^{1} P_{n} P_{m} d x=D_{n} \frac{2 n(n+1)}{2 n+1} \delta_{n m}
$$

The second term in Eq. (23) becomes.

$$
\int_{-1}^{1}\left(1-x^{2}\right) \sum_{m} D_{m} P_{m}^{\prime} \frac{d \beta U_{e}}{d x} P_{n} d x
$$

$$
\begin{aligned}
& \frac{d \beta U_{e}}{d x}=\eta x+\zeta \\
& \eta=-H_{k} \beta m, \quad \zeta=\left(H_{s}-H\right) \beta m
\end{aligned}
$$

$$
\left(1-x^{2}\right) P_{m}^{\prime}=m P_{m-1}-m x P_{m}
$$

$$
\sum_{m} D_{m} \int_{-1}^{1}\left(m P_{m-1}-m x P_{m}\right)(\eta x+\zeta) P_{n} d x
$$

$$
x P_{n}=\frac{1}{2 n+1}\left[(n+1) P_{n+1}+n P_{n-1}\right]
$$


Therefore, the matrix elements of the secular equation include two parts: the diagonal parts, Eq. (26); the diagonal and non-diagonal parts, Eq. (31).

It is noticed that the right side part of the secular equation Eq. (21) is dependent on $n$, so it is not the standard eigen-value equation $\mathrm{AX}=\mathrm{cX}$. Take the normalized Legendre functions $p_{m}(x)$ instead of $P_{m}(x)$,

$$
p_{m}(x)=\sqrt{\frac{2 n+1}{2}} P_{m}(x)
$$

so that.

$$
\int_{-1}^{1} p_{m}(x) p_{n}(x) d x=\delta_{m n}
$$

Using the normalized Legendre functions $p_{m}(x)$, it can obtain the secular equation $\mathrm{AX}=\mathrm{cX}$, the matrix elements of the equation, the diagonal term Eq. (25) becomes $n(n+1) \delta_{n m}$, and the non-diagonal term Eq. (31) becomes

$$
\begin{aligned}
& \frac{m(m+1)}{2 m+1}\left[\frac{\eta(n+1)}{2 n+1} \delta_{m-2, n}+\zeta \delta_{m-1, n}+\frac{\eta n}{2 n+1} \delta_{m, n}\right. \\
& \left.-\frac{\eta(n+1)}{2 n+1} \delta_{m, n}-\zeta \delta_{m+1, n}-\frac{\eta n}{2 n+1} \delta_{m+2, n}\right]
\end{aligned}
$$

\subsection{Secular Equation of Dimensionless form}

Multiplying the numerator and denominator of the eigenvalue $c$, Eq. (16) by $\left(H+H_{k}\right)$, and using.

$$
\frac{1}{\tau_{0}}=\alpha \gamma\left(H+H_{k}\right)
$$

we obtain.

$$
c=\lambda \tau_{0} \beta m\left(H+H_{k}\right)
$$

where $\tau_{0}$ is a time unit, $H$ and $H_{k}$ are the internal magnetic field and anisotropic magnetic field, respectively.

Divide the whole secular equation by.

$$
\xi=\beta m\left(H+H_{k}\right)
$$

the eigenvalue in the Eq. (17) becomes $c=\lambda \tau_{0}$, a dimensionless quantity.

In the new secular equation, the parameters become.

$$
\eta^{\prime}=-\frac{H_{k}}{H+H_{k}}, \quad \zeta^{\prime}=\frac{H_{s}-H}{H+H_{k}}=\frac{I}{I_{c}}-1
$$

Meantime, the diagonal term $n(n+1) \delta_{n m}$ should be divided by $\xi$, Eq. (37).

From the above discussion it found that the temperature relation is only included in the diagonal matrix elements, divided by $\xi$, Eq.(37). If we take $m=4 \pi \times 10^{-24} \mathrm{Vsm}, H=10^{6}$ $\mathrm{A} / \mathrm{m}, H_{k}=10^{4} \mathrm{~A} / \mathrm{m}$, then it can obtain $\xi=9.2 \times 10^{5} / T(\mathrm{~K})$.
Hence, the diagonal matrix elements are of $10^{-6} \times T$ order of magnitude, the effect of temperature is very small.

\subsection{Some Physical Quantities}

Magnetic moment $m=\mu_{0} M V$, where $\mu_{0}$ is the vacuum magnetic permeability, $M$ is the magnetization, and $V$ is the volume of the free layer. We take $M=10^{6} \mathrm{~A} / \mathrm{m}, V=10^{-23} \mathrm{~m}^{3}$, obtain $m=4 \pi \times 10^{-24} \mathrm{Vsm}$.

Spin torque field.

$$
H_{s}=\left(\frac{\hbar}{2 e}\right) \frac{I p}{\alpha m}
$$

where $I$ is the current driving the spin reverse, $p$ is the spin polarization coefficient, $\alpha$ is the damping coefficient. We take $I=1 \mathrm{~mA}=10^{-3} \mathrm{~A}, p=1, \alpha=0.02, m=4 \pi \times 10^{-24} \mathrm{Vsm}$ and using $\hbar / 2 e=3.28 \times 10^{-16} \mathrm{Vs}$, obtain $H_{s}=1.3 \times 10^{6} \mathrm{Am}^{-1}$.

Time unit, from Eq. (35), $1 / \tau_{0}=\alpha \chi\left(H+H_{k}\right)$. taking $H=10^{6}$ $\mathrm{Am}^{-1}, H_{k}=10^{4} \mathrm{Am}^{-1}, \alpha=0.02$, and using $\gamma=176 \mathrm{GHz} / \mathrm{T}$, obtain $1 / \tau_{0}=4.47 \mathrm{GHz}$, and $\tau_{0}=0.22 \mathrm{~ns}$.

Intrinsic threshold current.

$$
I_{c}=\left(\frac{2 e}{\hbar}\right)\left(\frac{\alpha}{p}\right) m\left(H+H_{k}\right)
$$

where $H_{k}$ is the internal anisotropic field $H_{k}=K / M_{s}<<H$. We take $p=1, \alpha=0.02, m=4 \pi \times 10^{-24} \mathrm{Vsm}$, obtain $I_{c}=0.766 \mathrm{~mA}$.

\subsection{Convergence of the Expansion Method}

There are input parameters: $\eta^{\prime}$ and $\zeta$ (Eq. (38)) and $1 / \xi$ (Eq. (37)). As a test, we take $\eta^{\prime}=-0.01$ and $\zeta^{\prime}=2.0,1 / \xi=0.01$, calculate the eigenvalues $\lambda \tau_{0}$ for different numbers of the expansion Legendre functions $\mathrm{N}$, the results are listed in Table 1.

Table 1. Eigenvalues as functions of the number of expansion basis $N$.

\begin{tabular}{llllll}
\hline $\mathbf{N}$ & $\mathbf{4 0}$ & $\mathbf{6 0}$ & $\mathbf{8 0}$ & $\mathbf{1 0 0}$ & $\mathbf{2 0 0}$ \\
\hline$\left(\lambda \tau_{0}\right)_{1}$ & 1.77373 & 1.86045 & 1.86045 & 1.86045 & 1.86045 \\
& 0.19432 & & & & \\
$\left(\lambda \tau_{0}\right)_{2}$ & 2.07068 & 2.10930 & 2.10930 & 2.10930 & 2.10930 \\
& 0.79618 & & & & \\
\hline
\end{tabular}

From Table 1 we see that:

1. The lowest eigenvalue is 1.86045 , which is in agreement with the value in Ref. [12]. When $\mathrm{N}=40$, the lowest two eigenvalues are complex, the second rows are their imagine parts.

2. When $\mathrm{N} \geq 60$, the two lowest eigenvalues are real, and become convergent to five decimal place. Afterward, we will take $\mathrm{N}=100$ in our calculation. In Ref. [12] they expand $F(x)$ via Taylor series, $F(x)=\Sigma_{n} a_{n} x^{n}$, the convergent results are obtained for larger $\mathrm{N}=300$.

3. The corresponding eigenvectors include about 50 lowest basic functions (Legendre functions) to five decimal places. 


\section{Calculation Results}

\subsection{Effect of $\left(I / I_{c}-1\right)$}

There are three parameters $\eta^{\prime}, \zeta$ (Eq. (38)), and $1 / \xi($ Eq. (37)), dependent on $H, H_{k}$, and $H_{s}\left(I / I_{c}-1\right)$. Take $\eta^{\prime}=-0.01,1 / \xi$ $=0.01 ; \eta^{\prime}=-0.01,1 / \xi=0.1 ;$ and $\eta^{\prime}=-0.1,1 / \xi=0.01$, and calculate the first and second eigenvalues as functions of $\zeta=$ $I / I_{c}-1$, the results are shown in Figs. 1 and 2, respectively.

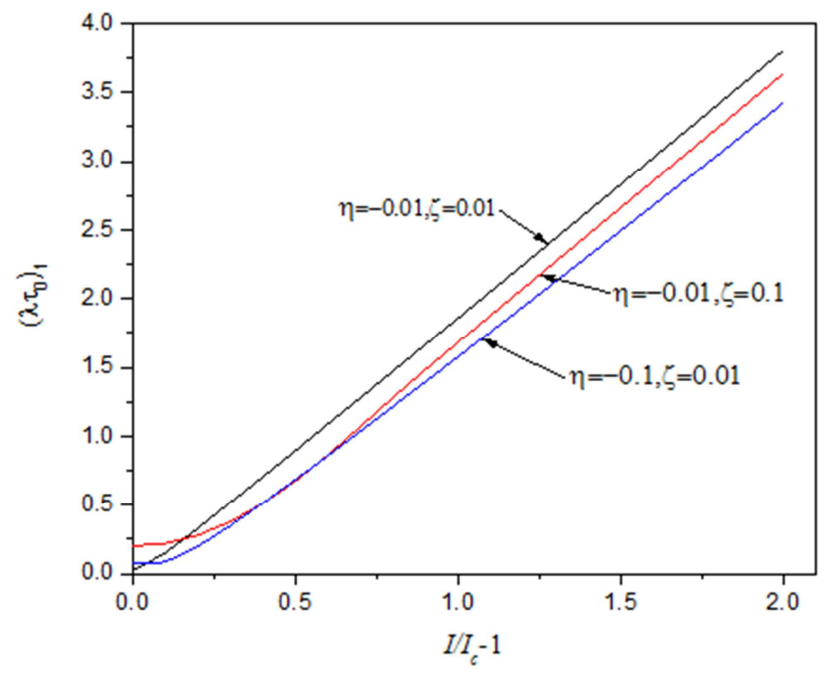

Figure 1. First eigenvalues $\left(\lambda \tau_{0}\right)_{1}$ as functions of $I / I_{c^{-}} 1$ for three cases: $\eta^{\prime}=$ $-0.01,1 / \xi=0.01 ; \eta^{\prime}=-0.01,1 / \xi=0.1 ;$ and $\eta^{\prime}=-0.1,1 / \xi=0.01$.

Figure 1 shows that the $\left(\lambda \tau_{0}\right)_{1}$ increase with $I / I_{c}-1$ linearly except near the zero. For different parameter $\eta^{\prime}$ and $1 / \xi$ there are difference between three curves, but the difference is small.

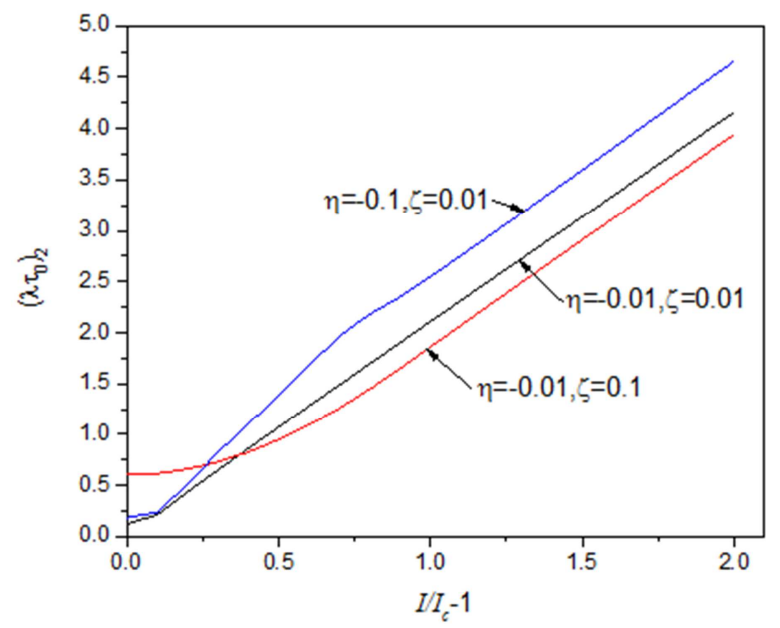

Figure 2. Second eigenvalues $\left(\lambda \tau_{0}\right)_{2}$ as functions of $I / I_{c}-1$ for three cases: $\eta^{\prime}=$ $-0.01,1 / \xi=0.01 ; \eta^{\prime}=-0.01,1 / \xi=0.1 ;$ and $\eta^{\prime}=-0.1,1 / \xi=0.01$.

Similar to $\left(\lambda \tau_{0}\right)_{1}$ (Figure 1$)$, the $\left(\lambda \tau_{0}\right)_{2}$ increase with $I / I_{c^{-}}-1$. It is noticed that the uppermost curve for $\eta^{\prime}=-0.1,1 / \xi=0.01$ is not smooth, because for $0.3 \leq I / I_{c}-1 \leq 0.7$, the $\left(\lambda \tau_{0}\right)_{2}$ becomes complex, Figure 2 only shows their real parts. It is not surprising that the eigenvalues and their corresponding eigenvectors of the secular equation with real and non-symmetric coefficients are possible to be real or complex.
It means that the probability density $P$ not only decays with time, but also oscillates with time if the eigenvalue is complex. In the region $0.3 \leq I / I_{c}-1 \leq 0.7$ the eigenvalues $\left(\lambda \tau_{0}\right)_{2}$ are listed in Table 2.

Table 2. The eigenvalues $\left(\lambda \tau_{0}\right)_{2}$ in the region $0.3 \leq I / I_{c}-1 \leq 0.7$.

\begin{tabular}{llllll}
\hline$I / I_{c^{-}} 1$ & 0.3 & 0.4 & 0.5 & 0.6 & 0.7 \\
\hline $\begin{array}{l}\text { Real } \\
\left(\lambda \tau_{0}\right)_{2}\end{array}$ & 0.82427 & 1.10233 & 1.38818 & 1.67779 & 0.96949 \\
$\begin{array}{l}\text { Imagine } \\
\left(\lambda \tau_{0}\right)_{2}\end{array}$ & 0.11294 & 0.16191 & 0.18160 & 0.17500 & 0.13333 \\
\hline
\end{tabular}

\subsection{Effect of $H_{k}$}

Fix the $I / I_{c}-1=0,0.5,1.0,1.5$, and 2.0 , and $1 / \xi=0.01$, calculate the eigenvalues $\left(\lambda \tau_{0}\right)_{1}$ and $\left(\lambda \tau_{0}\right)_{2}$ as functions of $\eta^{\prime}$, the results are shown in Figs. 3 and 4, respectively. From Eq. (28) we see that $\eta^{\prime}$ is proportional to $H_{k}$, if assuming that $H+H_{k}$ = constant.

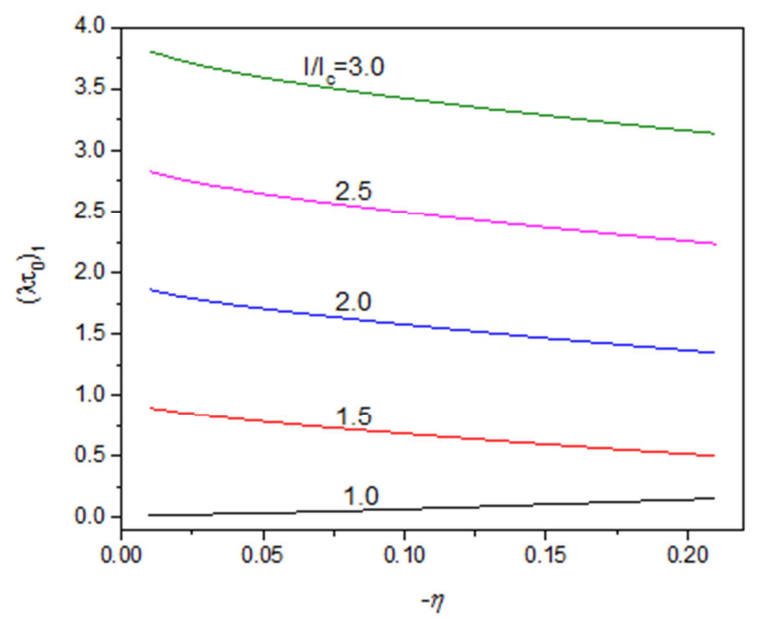

Figure 3. $\left(\lambda \tau_{0}\right)_{1}$ as functions of $-\eta^{\prime}$, for $I / I_{c^{-}}-1=0 ., 0.5,1.0,1.5$, and 2.0 , and $1 / \xi=0.01$.

From Figure 3 we see that $\left(\lambda \tau_{0}\right)_{1}$ decrease with $-\eta^{\prime}\left(H_{k}\right.$, if $H+H_{k}=$ const. $)$, except for $I / I_{c}=1.0$. The whole trend is in agreement with Fig. 1 in Ref. [12].

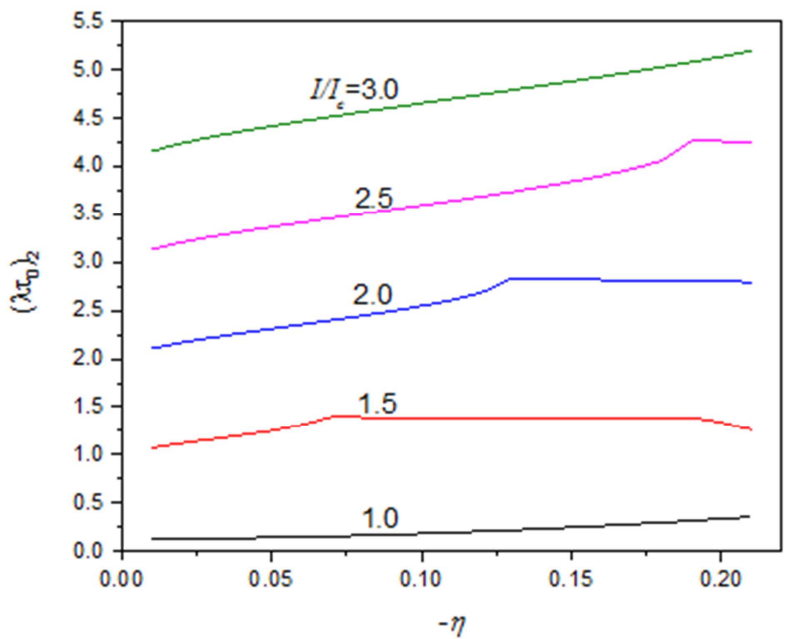

Figure 4. $\left(\lambda \tau_{0}\right)_{2}$ as functions of $-\eta^{\prime}$, for $I / I_{c^{-}}-1=0 ., 0.5,1.0,1.5$, and 2.0, and $1 / \xi=0.01$. 
From Figure 4 we see that $\left(\lambda \tau_{0}\right)_{2}$ increase with $-\eta^{\prime}\left(H_{k}\right.$, if $H+H_{k}=$ const.). Some curves are also not smooth, because the eigenvalues are complex, the curves only show the real parts of $\left(\lambda \tau_{0}\right)_{2}$. For $I / I_{c}=1.5$, the complex $\left(\lambda \tau_{0}\right)_{2}$ exists in the region $-0.07 \leq \eta^{\prime} \leq-0.19$. For $I / I_{c}=2.0$, the complex $\left(\lambda \tau_{0}\right)_{2}$ exists in the region $-0.13 \leq \eta^{\prime}$. For $I / I_{c}=2.5$, the complex $\left(\lambda \tau_{0}\right)_{2}$ exists in the region $-0.19 \leq \eta^{\prime}$.

\section{Summary}

We proposed a Legendre function expansion method to solve the simplified Fokker-Plank equation to study the dynamics of a macrospin under spin-torque-driven magnetic reversal at finite temperature. We obtained the first and second eigenvalues $\left(\lambda \tau_{0}\right)_{1}$ and $\left(\lambda \tau_{0}\right)_{2}$ as functions of $I / I_{c}$ and $H_{k}$. The Legendre function expansion method compared with the previous Taylor series expansion method has faster convergence properties and clear physical means. Besides, it is found that in some case, especially the second eigenvalue $\left(\lambda \tau_{0}\right)_{2}$ can become complex, that means that the probability density $P$ not only decays with time, but also oscillates with time.

\section{Acknowledgements}

This work was supported by the Advanced Research Plan of Chinese Academy of Sciences: QYZDY-SSW-JSC015.

\section{References}

[1] Chi-Feng Pai, Luqiao Liu, Y. Li, H. W. Tseng, D. C. Ralph, and R. A. Buhrman, "Spin transfer torque devices utilizing the giant spin Hall effect of tungsten," Appl. Phys. Lett. 101, 122404 (2012).

[2] Xiao-binWang, Yi-ran Chen, Hai-wen Xi, Hai Li, Dimitar Dimitrov, "Spintronic Memristor Through
Spin-Torque-Induced Magnetization Motion," IEEE ELECTRON DEVICE LETTERS, VOL. 30, NO. 3, MARCH 2009.

[3] J. Z. Sun, S. L. Brown, W. Chen, E. A. Delenia, "Spin-torque switching efficiency in $\mathrm{CoFeB}-\mathrm{MgO}$ based tunnel junctions," PHYSICAL REVIEW B 88, 104426 (2013).

[4] Xiao-bin Wang, Yuan-kai Zheng, Hai-wen Xi, and Dimitar Dimitrov, "Thermal fluctuation effects on spin torque induced switching: Mean and variations," J. Appl. Phys. 103, 034507 (2008).

[5] Xiao-bin Wang, H. Neal Bertram, and Vladimir L. Safonov, "Thermal-dynamic reversal of fine magnetic grains with arbitrary anisotropy axes orientation," Journal of Applied Physics 92, 2064 (2002)

[6] R. Bonina, G. Bertottia, C. Serpicob, I. D. Mayergo- yz, "Effect of thermal fluctuations in spin-torque driven magnetization dynamics," Journal of Magnetism and Magnetic Materials 316 (2007) e919-e922

[7] Xiao-bin Wang, Wen-zhong Zhu, and Dimitar Dimitrov, "Current fluctuations and magnetization dynamics symmetry in spin-torque-induced magnetization switching," PHYSICAL REVIEW B 78, 024417 (2008).

[8] D. V. Berkova, J. Miltat, "Spin-torque driven magnetization dynamics: Micromagnetic modeling," Journal of Magnetism and Magnetic Materials 320 (2008) 1238-1259.

[9] J. Z. Sun, R. P. Robertazzi, J. Nowak, et al. "Effect of subvolume excitation and pin-torque efficiency on magnetic switching," Phys. Rev. B 84, 064413 (2011).

[10] J. Z. Sun, Physical Principles of Spin Torque, in Handbook of Spintronics, Eds. Y. B. Xu, D. D. Awschalom, J. Nitta, Springer, 2016, Vol. IV, p. 1339.

[11] J. He, J. Z. Sun, S. Zhang, "Switching speed distribution of spin-torque-induced magnetic reversal," J. Appl. Phys. 101, 09A501 (2007).

[12] Zhu-Xi Wang, Dun-Ren Guo, Introduction of Special functions (in Chinese), Peking University Press, Beijing, 2010. 